\title{
Atuação e desafios da equipe de medicina e saúde do adolescente: percepções dos
}

\section{profissionais}

\author{
Performance and challenges of the adolescent medicine and health team: professionals' perceptions \\ Desempeño y retos del equipo de medicina y salud del adolescente: percepciones de los \\ profesionales
}

Recebido: 14/09/2021 | Revisado: 22/09/2021 | Aceito: 24/09/2021 | Publicado: 25/09/2021

\author{
Dalva Alves Silva \\ ORCID: https://orcid.org/0000-0001-7798-1087 \\ Universidade Federal de São Paulo, Brasil \\ E-mail: dalvaunifesp@gmail.com \\ Jeane Barros de Souza \\ ORCID: https://orcid.org/0000-0002-0512-9765 \\ Universidade Federal da Fronteira Sul, Brasil \\ E-mail: jeane.souza@uffs.edu.br \\ Maria Sylvia de Souza Vitalle \\ ORCID: https://orcid.org/0000-0001-9405-4250 \\ Universidade Federal de São Paulo, Brasil \\ E-mail: sylviavitalle@gmail.com
}

\begin{abstract}
Resumo
Este artigo tem como objetivo compreender e discutir como ocorre o atendimento em equipe de medicina e saúde do adolescente, nas percepções dos profissionais que se dedicam a esta forma de atuação. O método empregado foi pesquisa descritiva, de abordagem qualitativa, com a participação de 25 profissionais atuantes em cinco equipes de medicina e saúde do adolescente do Brasil. Realizamos uma entrevista semiestruturada e observação participante. A organização e análise dos dados ocorreu por meio da análise temática, da qual destacamos duas categorias: Atendimento à saúde dos adolescentes e Desafios do cotidiano das equipes de medicina e saúde do adolescente. Encontramos profissionais altamente preparados para o atendimento diferenciado aos adolescentes em todo ciclo etário. Se apresentaram inúmeros desafios estruturais relativos à constituição de equipe, desde a extensão dos atendimentos intersetoriais até a necessidade de trabalho colaborativos e formativos, que promovam trocas recíprocas entre profissionais da saúde e equipe escolar e que, portanto, reverberem na expansão, qualidade e acesso dos adolescentes aos serviços próprios para a faixa etária em que se encontram. No tocante à formação dos profissionais emergem questões de comunicação, teóricas e práticas, históricoculturais. Concluímos que, em que pese a existência de muitos desafios para a atuação destes profissionais, eles percebem que há necessidade de políticas públicas que efetivem as garantias de direitos e há um esforço permanente para a superação destes desafios.
\end{abstract}

Palavras-chave: Equipe multiprofissional; Escola; Medicina do adolescente; Saúde do adolescente; Serviços de saúde.

\begin{abstract}
This article aims to understand and discuss how care in a medical and adolescent health team occurs, in the perceptions of professionals who are dedicated to this form of action. The method used was descriptive research, with a qualitative approach, with the participation of 25 professionals working in five medical and adolescent health teams in Brazil. We conducted a semi-structured interview with each participant, using participant observation. Data were organized and analyzed through thematic analysis, from which two categories stand out: Adolescent health care and Daily challenges for medical and adolescent health teams. We find professionals who are highly prepared to provide differentiated assistance to teenagers across all age groups. Numerous structural challenges related to team building were presented, from the extension of intersectoral care to the need for collaborative and training work, which promote reciprocal exchanges between health professionals and school staff and, therefore, reverberate in the expansion, quality and access of adolescents to services suitable for their age group. With regard to the training of professionals, issues of communication, theoretical and practical, historical and cultural context. We conclude that, despite the existence of many challenges for the performance of these professionals, they realize that there is a need for public policies that enforce the guarantees of rights and there is a permanent effort to overcome these challenges.
\end{abstract}

Keywords: Patient care team; Schools; Adolescent medicine; Adolescent health; Health services.

\section{Resumen}

Este artículo tiene como objetivo comprender y discutir cómo se lleva a cabo el atendimiento en equipo de la medicina y salud del adolescente, según las percepciones de los profesionales que se dedican a esta forma de trabajo. El método utilizado fue la investigación descriptiva, con enfoque cualitativo, con la participación de 25 profesionales que trabajan en cinco equipos de medicina y salud del adolescente en Brasil. Realizamos una entrevista semiestructurada con cada participante, mediante observación participativa. La organización y análisis de los datos se llevó a cabo a través de un 
análisis temático, del cual se destacan dos categorías: Atendimiento a la salud del adolescente y Desafíos diarios para los equipos de la medicina y de salud del adolescente. Encontramos profesionales altamente preparados para brindar asistencia diferenciada a adolescentes de todas las edades. Se presentaron numerosos desafíos estructurales relacionados con la construcción de equipos, desde la extensión de la atención intersectorial hasta la necesidad del trabajo colaborativo y formativo, que promueven intercambios recíprocos entre los profesionales de la salud y el personal escolar y, por tanto, repercuten en la expansión, calidad y acceso de los adolescentes a servicios adecuados para su grupo de edad. En lo que respecta a la formación de profesionales, surgen cuestiones de comunicación, teóricas y prácticas, históricas y culturales. Concluimos que, a pesar de la existencia de muchos desafíos para el desempeño de estos profesionales, se dan cuenta de que existe una necesidad de políticas públicas que hagan valer las garantías de derechos y existe un esfuerzo permanente por superar estos desafíos.

Palabras clave: Equipo multiprofesional; Instituciones Académicas; Medicina del adolescente; Salud del adolescente; Servicios de salud.

\section{Introdução}

A medicina de adolescente é uma área específica cada vez mais divulgada no meio acadêmico e nas redes sociais, mas ainda pouco conhecida pela população em geral. Historicamente, entre meados das décadas de 1970 e 1990 , os serviços de medicina e de atenção à saúde de adolescentes, que já vinham atuando de maneira mais isolada, foram oficialmente formalizados no Brasil (Queiroz et al., 2013).

Em termos de aplicação, circunscrita ao âmbito da pediatria, a medicina do adolescente delimitou a atuação profissional a partir da exigência de titulação para esse atendimento (Conselho Federal de Medicina - Resolução $\mathrm{N}^{\circ}$ 1634/2002). Enquanto o serviço de saúde do adolescente foi se constituindo no âmbito da Saúde Coletiva, com o modelo de atuação em equipe multidisciplinar, integrando ações de diversas áreas, mais voltado às populações e à atenção integral dos adolescentes (Queiroz et al., 2013). Entretanto, tanto a medicina de adolescentes como a saúde de adolescentes marcaram duas formas de atuação profissional, ambas visando ao atendimento integral e constituindo-se dentro de uma estrutura de trabalho em equipe multiprofissional.

A atuação em equipe de atendimento em medicina e saúde de adolescente é um tema que tem sido discutido há mais de quatro décadas, quando os principais marcos legais de proteção e garantias dos direitos de crianças e adolescentes foram sendo constituídos e promulgados e os programas e serviços de atendimento à esta população foram sendo formalizados.

Um dos pioneiros no atendimento integral à saúde dos adolescentes, Juckowisky Filho (1991), já destacava como necessárias as ações em equipe voltadas à população de adolescentes, uma vez que um profissional sozinho, ainda que altamente preparado, encontraria limites em sua atuação, em razão das questões apresentadas nas consultas, que muitas vezes supera(va)m as questões médicas e saberes instrumentais, necessitando um olhar mais abrangente também para as questões psicossociais, de valores humanos e espirituais.

A adolescência tem sido comumente definida por critérios cronológicos. A Organização Mundial de Saúde (OMS, 1977) a define como uma fase da vida humana que vai dos 10 anos aos 20 anos incompletos. Este critério também é adotado pelo Ministério da Saúde do Brasil (2018). O Estatuto da Criança e do Adolescente (ECA, 1990), estabelece que a adolescência compreende o período de vida entre 12 e 18 anos. Todavia, o Estatuto da Juventude estabelece a idade dos 15 aos 24 anos, e desse modo, aqueles com idade acima de 15 anos se encontram [também] incluídos na definição cronológica de juventude como "pessoas jovens"; termo este utilizado para o grupo de adolescentes que se encontram no período intermediário dos 15 aos 20 anos de vida (Estatuto da Juventude, 2013; OPAS, 2019).

Embora o estabelecimento de critérios cronológicos para definir adolescência e juventude permita maior objetividade para a elaboração e proposição de políticas públicas voltadas a esta população, ele não é abrangente o suficiente, uma vez que não abarca toda a complexidade no contexto sócio-histórico-cultural (Peres e Rosenburg, 1998; Fernandes e Santos, 2020) Além disso, importante considerar dentre os aspectos sócio-histórico-culturais, a questão da geração enquanto categoria de análise, pois ela é extremamente relevante na análise do processo saúde-adoecimento, ainda que na atualidade surja de forma 
pouco explorada nos estudos pertinentes ao campo da saúde, sendo de forma geral, reduzida ao estudo de faixas ou coortes etárias (Costa Júnior \& Couto, 2015).

Em termos de política pública, o Programa de Saúde do Adolescente (PROSAD/1989), foi “o primeiro a se preocupar de forma específica com a saúde dos adolescentes, o que representou um avanço em termos de saúde pública destinada a este público (Jager et al., 2014, p. 212)”, mostrando-se em conformidade com o Sistema Único de Saúde (SUS), com ações embasadas nos princípios de equidade, resolubilidade, acessibilidade e integralidade, considerando a atuação em equipe de saúde como uma forma de efetivar as suas ações.

O ECA, é um marco legal, regulatório, que tem como objetivo a proteção integral de crianças e adolescentes. Suas normas estabelecem crianças e adolescentes como sujeitos de direito, bem como de deveres que envolvem as responsabilidades e competências das famílias, da sociedade e suas instituições.

As Diretrizes Nacionais de Atenção à Saúde de Adolescentes e Jovens (2010), reafirmam o atendimento integral a crianças, adolescentes e jovens no âmbito do SUS, assegurado pelo ECA, que garante o acesso universal e igualitário às ações e serviços envolvendo a promoção, proteção e recuperação da saúde, com reconhecimento da vulnerabilidade do grupo jovem com idades entre 15 e 24 anos.

Tanto o ECA como as diretrizes do SUS, contemplam a atuação em equipe multi e interdisciplinar. O SUS a aborda na perspectiva da Estratégia de Saúde da Família (ESF), como a maneira de atuação indicada ao atendimento em saúde do adolescente, ao suporte familiar, assim como para as avaliações das diversas ocorrências e relatórios que oferecem suporte às decisões judiciais que tratam de questões envolvendo crianças e adolescentes, cujas condições de vida, saúde e bem-estar estão comprometidas, necessitando, portanto, de proteção.

Considerando os diferentes contextos e condições de vida e acesso dos adolescentes e suas famílias a bens e serviços fundamentais como direito humano, assim como as múltiplas questões trazidas por eles às consultas, o atendimento oferecido implica na necessidade de profissionais com habilidades específicas de escuta, acolhimento e atenção diferenciada para atendêlos. Contudo, na composição ideal de equipes multi/interdisciplinares, não se encontra como necessário à equipe o profissional de saúde especializado em atendimento aos adolescentes.

Diante do exposto, questiona-se: como ocorre o atendimento em equipe de medicina e saúde do adolescente a partir do ponto de vista de profissionais que se dedicam a esta forma de atuação? Este estudo se justifica a fim de dar maior visibilidade aos serviços especializados em saúde do adolescente no Brasil, com ênfase no atendimento prestado e nos desafios das equipes de atuação, buscando ampliação de políticas públicas voltadas ao público adolescente, no âmbito da saúde e da educação. Logo, este estudo teve como objetivo compreender e discutir como ocorre o atendimento em equipe de medicina e saúde do adolescente nas percepções dos profissionais que se dedicam a esta forma de atuação.

\section{Metodologia}

Trata-se de um estudo descritivo, de abordagem qualitativa, que permite uma melhor interpretação dos fenômenos a partir de seus significados e contextos, proporcionando uma visão abrangente dos problemas (GIL, 2017), realizado no período de janeiro de 2015 a junho de 2017. Assim, a abordagem qualitativa tornou-se o percurso metodológico indicado, uma vez que esta privilegia compreender sobre o atendimento em equipe de medicina e saúde do adolescente a partir do olhar dos próprios profissionais.

Para organizar os participantes do estudo, realizou-se um levantamento dos Hospitais Universitários (HU), via Google, para a busca dos contatos das coordenações das equipes de Medicina e Saúde do Adolescente, atuantes em ambulatório hospitalar no Brasil. A partir de então, realizou-se os contatos com os profissionais, convidando-os a integrar o estudo. 
Os coordenadores das equipes que aceitaram o convite em participar do estudo emitiram uma Notificação de Aceite para a realização da pesquisa no local. A partir do aceite, os profissionais das equipes foram convidados a participar, via chefia/coordenação, e os que aceitaram o fizeram voluntariamente, assinando o Termo de Consentimento Livre e Esclarecido (TCLE).

O estudo contou com a participação de 25 profissionais atuantes em cinco equipes de medicina e saúde do adolescente do Brasil. Como critério de inclusão considerou-se: ser profissional integrante de uma equipe multidisciplinar/multiprofissional de atendimento aos adolescentes, atuante em ambulatório de HU. Este critério foi adotado por estarem os HU e seus ambulatórios ligados ao ensino, pesquisa e extensão assistencial, tripé que sustenta a formação universitária. Como critério de exclusão considerou-se aqueles que estavam afastados do serviço no período da coleta de dados.

Os profissionais das equipes foram entrevistados em seus respectivos serviços de atendimento de saúde ao adolescente, localizados no Sul (Santa Catarina), Sudeste (São Paulo e Rio de Janeiro), Centro-oeste (Distrito Federal) e Nordeste (Pernambuco).

Para a coleta de dados utilizamos a entrevista semiestruturada, que foi conduzida pela pesquisadora principal, primeira autora deste artigo, permitindo elaborar um roteiro de questões previamente determinadas, mas ao mesmo tempo com abertura para os entrevistados terem a liberdade de responder "abrindo-se a novos assuntos que não estavam previstos nas questões principais" (Moreira, 2002, p.55). A entrevista continha questões sobre a atuação na medicina e saúde do adolescente e os desafios do trabalho em equipe na assistência ao adolescente.

As entrevistas tiveram duração de aproximadamente 60 minutos e foram gravadas com a devida autorização dos participantes. Além das entrevistas, manteve-se a técnica da observação participante, empregada para buscar entender como os participantes viam a sua atuação em equipe e o que tinham a dizer a partir de seus respectivos contextos de atuação. Esta escolha possibilitou ouvir os profissionais, de modo a compreender e interpretar suas falas e assim conhecer as equipes das quais faziam parte, "a partir das perspectivas dos próprios sujeitos sob estudo" (Paul, 2013, p.190).

A organização e análise dos dados ocorreu por meio da análise temática, proposta por Bardin (2016), em que seguiuse as seguintes etapas: 1) transcrição de todo material coletado nas entrevistas e encaminhamento a cada entrevistado(a) para conhecimento, ajustes, aceite e devolução às pesquisadoras; 2) realização de uma pré-análise que consistiu de nova leitura dos dados; 3) exploração do material de análise, por meio de recortes dos eixos temáticos; 4) realização de um trabalho cuidadoso de leitura e releitura do material coletado, com atualização a partir da observação de novos conteúdos que puderam contribuir como material de análise; 5) estabelecimento de duas categorias.

Essa pesquisa obteve a aprovação do Comitê de Ética e Pesquisa de uma Universidade Federal de São Paulo, com o CEP/UNIFESP n ${ }^{\circ}$. 0427/2014 e Parecer nº. 921.362, de 16/12/2014, estando em conformidade com a Resolução No 466/2012 do Conselho Nacional de Saúde, que regulamenta as pesquisas envolvendo seres humanos. Para preservar o anonimato dos participantes, optou-se por representá-los pela sua profissão e equipe de atuação, sendo que foram enumeradas conforme a ordem das entrevistas realizadas em cada localidade: São Paulo foi considerado equipe 1 (Eq1), Distrito Federal equipe 2 (Eq2), Pernambuco equipe 3 (Eq3), Rio de Janeiro equipe 4 (Eq4) e Santa Catarina equipe 5 (Eq5).

Este artigo é produto da tese de doutorado da primeira autora (defendida e registrada em Ata de 30/06/2017/Campus Guarulhos/Unifesp).

\section{Resultados e Discussão}

Os 25 participantes do estudo integravam cinco diferentes equipes de atuação em saúde do adolescente no Brasil, conforme o Quadro 1, que retrata a caracterização de acordo com idade, sexo, instituição/local, atuação na equipe, titulação e religião. 
Quadro 1. Apresentação dos profissionais entrevistados para o

\begin{tabular}{|c|c|c|c|c|c|c|}
\hline 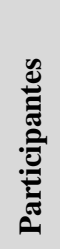 & 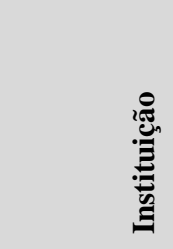 & 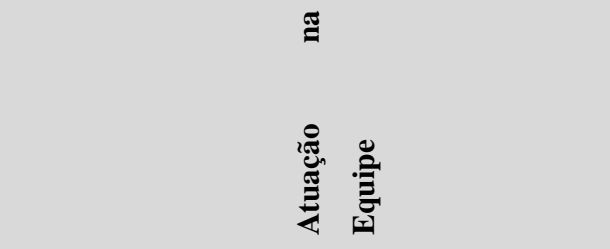 & 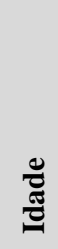 & 离 & 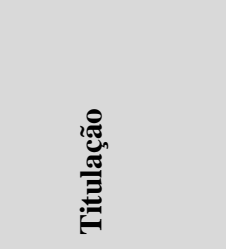 & 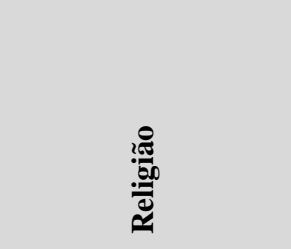 \\
\hline 01 & $\begin{array}{l}\text { EQUIPE } 1 \\
\text { SP }\end{array}$ & Prof $^{\mathrm{a}}$. Dra ${ }^{\mathrm{a}}$. Médica de Adolescentes & 65 & $\mathrm{~F}$ & $\begin{array}{l}\text { Doutorado } \\
\text { Livre-Docência }\end{array}$ & Israelita \\
\hline 02 & & Prof $^{\mathrm{a}}$. Dra . Médica de Adolescentes & 41 & $\mathrm{~F}$ & Doutorado & Católica \\
\hline 01 & & Prof $^{\mathrm{a}}$. Dr ${ }^{\mathrm{a}}$. Médica de Adolescentes & 62 & $\mathrm{~F}$ & $\begin{array}{l}\text { Doutorado } \\
\text { Pós-Doutorado }\end{array}$ & Católica/Espiritualista \\
\hline 02 & & Prof $^{\mathrm{a}}$. M . Médica Pediatra & 49 & $\mathrm{~F}$ & Mestrado & Católica/Budista \\
\hline 03 & $\begin{array}{l}\text { EQUIPE } 2 \\
\text { DF }\end{array}$ & $\begin{array}{l}\text { Prof. Dr. Neuropediatra especializado em } \\
\text { adolescentes }\end{array}$ & 59 & $\mathrm{M}$ & Doutorado & Evangélico \\
\hline 04 & & Médico Psiquiatra de crianças e adolescentes & 37 & M & Especialização & Não tem religião \\
\hline 05 & & Psicóloga & 55 & $\mathrm{~F}$ & Especialização & Católica/Espírita \\
\hline 01 & EQUIPE 3 & Prof $^{\mathrm{a}}$. Dr ${ }^{\mathrm{a}}$. Médica de Adolescentes & 66 & $\mathrm{~F}$ & Doutorado & Católica \\
\hline 02 & $\mathbf{P E}$ & Prof $^{\mathrm{a}}$. Dra ${ }^{\mathrm{a}}$. Médica de Adolescentes & 67 & $\mathrm{~F}$ & Doutorado & Católica \\
\hline 01 & & Prof. Dr. Médico Clínico de adolescentes & 66 & M & $\begin{array}{l}\text { Doutorado } \\
\text { Prof. Titular }\end{array}$ & Agnóstico \\
\hline 02 & & Prof $^{\mathrm{a}}$. Dra ${ }^{\mathrm{a}}$. Médica de Adolescentes & 66 & $\mathrm{~F}$ & Doutorado & Judaica \\
\hline 03 & & Médica de Adolescentes & 35 & $\mathrm{~F}$ & Mestrado & Católica \\
\hline 04 & & Serviço Social & 55 & $\mathrm{~F}$ & Mestrado & Evangélica \\
\hline 05 & EQUIPE 4 & Serviço Social & 50 & $\mathrm{~F}$ & Mestrado & Espírita \\
\hline 06 & RJ & Enfermeira & 53 & $\mathrm{~F}$ & Especialização & Espírita \\
\hline 07 & & Nutricionista & 43 & $\mathrm{~F}$ & Mestrado & Cristã \\
\hline 08 & & Psicóloga & 56 & $\mathrm{~F}$ & Mestrado & Protestante \\
\hline 09 & & Fisioterapeuta & 56 & $\mathrm{~F}$ & Especialização & Hindu/Yoga \\
\hline 10 & & Bibliotecária & 50 & $\mathrm{~F}$ & Graduação & Católica \\
\hline 11 & & Jornalista Científico & 41 & $\mathrm{M}$ & Especialização & Espírita Kardecista \\
\hline 01 & & Prof. Dr. Médico Pediatra & 51 & $\mathrm{M}$ & Doutorado & Católica \\
\hline 02 & & Psicóloga & 40 & $\mathrm{~F}$ & Especialização & Espiritualista \\
\hline 03 & EQUIPE 5 & Pedagoga & 53 & $\mathrm{~F}$ & Especialização & Católica \\
\hline 04 & SC & Pedagoga & 50 & $\mathrm{~F}$ & Especialização & Nenhuma \\
\hline 05 & & Pedagoga/Psicopedagoga & 56 & $\mathrm{~F}$ & Especialização & Cristã \\
\hline
\end{tabular}

estudo.

Quadro elaborado pelas autoras. 
Da análise de conteúdo das entrevistas duas categorias, que respondem ao objetivo do trabalho, são abordadas neste artigo: a) Atendimento à saúde dos adolescentes; b) Desafios do cotidiano das equipes de medicina e saúde do adolescente.

\subsection{Atendimento à saúde dos adolescentes}

As cinco equipes entrevistadas tiveram origem nas universidades, nos cursos de medicina, entre meados dos anos $70 \mathrm{e}$ final dos anos 90, nos departamentos e/ou divisão de pediatria, bem como na clínica médica, sendo todas inseridas em disciplinas voltadas à adolescência ou à infância e adolescência, constituindo-se desde o início por profissionais de diferentes formações.

$\mathrm{Na}$ constituição das equipes entrevistadas, independentemente do número de profissionais fixos em sua composição, pelo menos em parte, contavam com a participação de alunos do curso de medicina, de residentes em pediatria, bolsistas da pós-graduação, especialização, mestrado e/ou doutorado, de diferentes áreas de conhecimento, todos com interesses motivados pelo ensino, pesquisa e extensão assistencial em saúde da criança e do adolescente.

De acordo com os coordenadores das equipes, adolescentes com idades entre 10/12 e 18/20 anos, em primeira consulta, encontravam portas abertas. Em geral, eram atendidos pelo profissional médico que os ouvia, examinava, avaliava e fazia os devidos encaminhamentos dentro da equipe. A equipe de SC, diferentemente das demais, recebia estudantes vindos dos dois ciclos do Ensino Fundamental (idades de 6 a 14 anos), via Programa de Saúde na Escola, e via demanda espontânea, realizando em equipe as triagens de primeiro atendimento, quando médicos e psicólogas recebiam os pais ou responsáveis e as pedagogas e fonoaudiólogas atendiam as crianças e adolescentes, reunindo-se ao final e discutindo os encaminhamentos das avaliações e orientações.

Por se tratar de atendimento ambulatorial para adolescentes, instalados em HUs de referência nacional, as demandas eram grandes e muitos adolescentes e suas famílias quando chegavam nos serviços já tinham percorrido um longo caminho pelos serviços de saúde oferecidos principalmente pela rede pública. Nos principais motivos de consulta encontravam-se as queixas clínicas de saúde geral, bem como de ordem nutricional, postural, saúde mental, questões de gênero, questões escolares, violências de diferentes formas, incluindo casos de bullying, doenças crônicas, dentre outros.

Eisenstein (2018) considera que a medicina e saúde dos adolescentes abrangem aspectos diversos "associados em oito faces: proteção social, promoção da saúde, prevenção de riscos, rastreamento de doenças, diagnóstico, tratamento, reabilitação e reintegração social (p.22)". De modo que seria importante, necessariamente, que os profissionais, na medida de suas motivações e vínculos empregatícios buscassem por formação frente aos desafios com os quais vão se deparar, pois é preciso "entender a adolescência para melhor atender os adolescentes (p.22)", a exemplo do que nos conta o profissional:

Quando eu fui convidado para vir para o ambulatório de adolescência, eu disse - Eu vou, só que tenho que ter um treinamento em adolescência, num serviço de referência, [a coordenadora] conseguiu que eu fosse para Los Angeles e fiquei lá no Children's Hospital, em um serviço de adolescentes. Aí eu cheguei e já fui incluído na equipe de adolescência e estou aqui até hoje (Neuropediatra-Especializado em Adolescentes-Eq2).

No Brasil, foi estabelecida pelo Conselho Federal de Medicina a titulação em Medicina do Adolescente, como área de atuação da pediatria, mediante prova de título (CFM No 1634/2002), e recentemente, a Comissão Nacional de Residência Médica, na sua Resolução 51, publicada no Diário Oficial da União, em 02 de setembro de 2021, aprova a Medicina do Adolescente como nova matriz de competência do Programa de Residência Médica, fortalecendo a formação profissional para o atendimento dedicado aos adolescentes. Contudo, muitas dúvidas e questionamentos persistem a respeito de quem deve 
atendê-los. Neste sentido, embora os adolescentes tenham, em tese, seus direitos à vida e à saúde assegurados, ainda são colocados no atendimento pediátrico infantil e/ou no atendimento clínico de adultos, conforme os relatos a seguir:

É muito difícil, ele fica num limbo. Porque esses serviços são estruturados para a pediatria e para os adultos e aí nesse período entre 12 e 18 a coisa fica meio... tá, quem cuida? (Médica de Adolescentes7-Eq4). - Eu comecei a me inquietar como pediatra, porque eu não sabia nada de adolescência, ninguém nunca tinha falado o que era o adolescente, eu era professora e pediatra e via que na fila para o clínico tinha umas pessoinhas que não eram adultos ainda e estavam lá (Médica de Adolescentes4-Eq3).

Fernandes e Santos (2020) encontraram em seu estudo que, tanto para os gestores quanto para os profissionais, os cursos de educação permanente contribuem para qualificar os profissionais de saúde e para valorizá-los enquanto trabalhadores, no entanto, os cursos não têm contemplado o cuidado e os temas voltados à saúde de adolescentes.

De acordo com Azevedo (2019), “existe uma deficiência no atendimento aos adolescentes no contexto da saúde pública, uma vez que os programas existentes não são executados em sua totalidade (p.24)", quer seja pela falta de capacitação e formação acadêmica dos profissionais ou mesmo pela deficiência física nas unidades de atendimento, entre outros fatores, como o entendimento incompleto por causa da ambivalência de critérios distintos presentes nas legislações para um mesmo tema:

O Ministério diz para adotarmos o que a OMS e a OPAS dizem, que a adolescência vai dos 10 aos 20 anos. No ano seguinte sai o Estatuto (ECA) em que adolescente é dos 12 aos 18 anos. Isso é uma polêmica dentro do próprio país. Você está trabalhando com dois critérios diferentes (Médica de Adolescentes5-Eq3).

As considerações feitas pela médica são importantes, pois incidem em implicações legais e administrativas tanto para a obtenção de recursos para projetos e políticas públicas como para ações cotidianas referentes à própria oferta de serviços, uma vez que, de certa forma, dificultam o entendimento da importância de que os serviços de saúde deem ênfase ao atendimento especializado e integral aos adolescentes com idades dos 10 aos 19/20 anos, sem exceção, considerando as especificidades deste período do ciclo vital, conforme os relatos a seguir:

A rede só está atendendo até doze anos. Esse ambulatório aqui é muito importante porque é um ambulatório que atende, mas se formos pensar em dois milhões, que é a população de Brasília, se ampliarmos para o entorno, nós teremos quatro milhões de habitantes, é nada! É um “cheiro”, mas é alguma coisa (Médica de Adolescentes3-Eq2). A gente trabalha com muitas limitações nesse sentido, de determinados exames que você sabe que são importantes e que você não pode fazer porque o hospital não oferece; determinadas especialidades que você tem dificuldade de encaminhar o paciente porque não tem vaga... ou às vezes tem vaga, mas não tem vaga para adolescente. Essa é uma questão muito pulsante, a coisa do acesso do adolescente aos serviços de saúde (Médica de Adolescentes7-Eq4). - No atendimento individual dos ambulatórios e nesse trabalho, acho que o grande desafio são as políticas públicas. O serviço social trabalha com garantias de direitos, sem políticas públicas não tem serviço social, e esse é o grande limite (Assistente Social2- Eq4). - Para mim o desafio maior, que está claríssimo para mim, não tenho dúvidas do que eu vou te afirmar, é a integralidade das políticas públicas! Então, atendo o menino super bem aqui, mas ele precisa voltar para o contexto sociocomunitário e ter garantidas algumas questões mínimas de qualidade de vida, como condições de habitação, entendeu? Condições de acesso à água. Tem famílias que não tem água dentro de casa 
ainda! E aí, como eu faço com o menino portador de uma doença grave? A própria nutricionista aponta isso... mas esse menino não tem água em casa! E aí, como você contar com a rede pública para garantir saúde como direito de todos e saúde no seu sentido ampliado? (Assistente Social1-Eq4).

As políticas públicas voltadas à atenção integral de adolescentes são fundamentais para garantir que os adolescentes recebam atendimento em diferentes níveis de complexidade e tenham condições de acesso a bens e serviços que permitam seguir as orientações para sua saúde. As políticas públicas visam a propor ações dentro de uma abordagem sistêmica, indo além da visão estritamente voltada à prevenção de doenças e agravos, que levem em conta o ser humano em crescimento que busca por cuidados para saúde, além da oportunidade de poder ser ouvido e dar vasão a seus sonhos, espiritualidade e projetos de vida que repercutirão no presente e no futuro (Azevedo, 2019).

Com frequência, na fala dos entrevistados, a questão escolar mostrou-se como uma das grandes preocupações dos profissionais, na medida em que, por ultrapassarem seus campos de atuação, sentiam-se impotentes para lidar com dificuldades apresentadas pelos adolescentes. Mas como cidadãos, cientes da importância e do impacto da escolarização na vida presente e na vida futura dos adolescentes, sentiam-se tocados diante do que fazer:

Às vezes consultamos adolescentes do Ensino Médio que são analfabetos, analfabetos funcionais e não temos como resolver (Médica de Adolescentes1 e Médica de Aldolescentes2-Eq1). - A demanda escolar a gente não dá conta (Médica de Adolescentes3-Eq2). - A gente vê muitas crianças com problemas de aprendizagem que vão ser diagnosticadas, mas elas vêm tardiamente, com 11, 12, 13, 14 anos. Aí vão descobrir... ou a escola deixa para resolver lá no quinto, sexto ou sétimo ano quando começam com os problemas de comportamento, com o bullying, com embotamento acadêmico, o retraimento.... Nessa faixa etária adolescente a gente percebe mais a questão psicossocial (Pedagoga1-Eq5). - A nossa demanda é bem heterogênea, tem um número muito grande de crianças com problemas emocionais afetando a aprendizagem. (Psicóloga3-Eq5). - A gente já tinha um trabalho aqui com dois grupos de adolescentes com o mesmo questionário e com perguntas sobre as relações familiares e coisas desse tipo. O mais interessante é que nos dois lugares, os adolescentes respondiam 'qual é a pessoa que você tem como ...?' Primeiro era família; segundo, o namorado ou namorada; depois amigos e só em quarto lugar era a escola [...] Os resultados praticamente os mesmos. Se mudassem os gráficos... então eles tinham o respeito da família, dos amigos e daqueles que já, bem ou mal, começavam a brincar de relacionamentos com escolhas e o que me preocupou era que a Escola era o quarto lugar! A maioria deles não estava na escola, e quem estava, estava defasado (Médico Clínico de Adolescentes-Eq4).

A possibilidade de as equipes contarem com a cooperação entre saúde e educação constituindo-se como uma rede de apoio recíproco mostra-se muito favorável à integralidade do atendimento oferecido aos adolescentes. O Programa Saúde na Escola (PSE), criado em 2007, conforme nos mostram Moraes e Vitalle (2021) "é uma forma de atuação Intersetorial cuja atuação na saúde utiliza a escola como lócus da demanda de atendimento e a educação com ações preventivas e educativas (p.51)". Da mesma forma a equipe escolar pode ter oportunidades de ser recebida no ambiente em que os adolescentes e suas famílias buscam atendimento e orientações para sua saúde.

Em relação às questões escolares Schoen e Marteleto (2019) consideram importante que os professores busquem conhecimentos a respeito dos transtornos do neurodesenvolvimento, a exemplo do Transtorno de Déficit de Atenção e Hiperatividade (TDAH), bem como conhecimentos a respeito dos transtornos de aprendizagem, tendo em vista que muitos dos problemas escolares, decorrentes desses transtornos, se manifestam especialmente na adolescência. Importa, também, saber 
sobre as doenças crônicas, que podem afetar profundamente o adolescente, incidindo sobre seu desempenho escolar, problemas comportamentais, emocionais e de socialização. A parceria entre equipe de saúde e equipe escolar poderá beneficiar a todos: os profissionais, os adolescentes e suas famílias.

Assim observamos que a equipe de Santa Catarina (Equipe 5), por meio de parceria com escolas municipais, via PSE, desenvolveu grande expertise para a avaliação das dificuldades de aprendizagem, podendo contar na equipe com a participação de profissionais da área da educação (pedagogia e psicopedagogia) e com o compartilhamento entre setores - ambulatorial e escolar.

\subsection{Desafios do cotidiano das equipes de medicina e saúde do adolescente}

O SUS trouxe uma nova concepção de cuidado, empregando modelos de atendimento centrados na promoção de saúde integral, considerando as várias dimensões de vida dos indivíduos, os diferentes contextos e os determinantes sociais de saúde (Moraes \& Vitalle, 2021).

Diante dessa concepção de saúde integral, o trabalho em equipe não apenas se fortaleceu como tornou-se necessário, abrindo um importante espaço para o trabalho colaborativo, o manejo de novas tecnologias em saúde, um olhar para a prevenção de doenças e orientação profilática, tendo em conta os diferentes contextos e estilos de vida das pessoas que buscam pelos serviços. Desse modo, evidenciou-se a necessidade de mudanças no currículo acadêmico dos cursos de graduação na área da saúde (Moraes \& Vitalle, 2021).

Uma renovação curricular do curso de Medicina pode ser encontrada nas Diretrizes Curriculares Nacionais de 2014 que têm orientado para a formação mais flexível e abrangente desde a graduação, por meio das interações entre as diferentes áreas de conhecimentos com ênfase na formação para o trabalho em equipe, com práticas voltadas à promoção da comunicação entre profissionais e entre estes com os usuários e suas famílias, bem como com as comunidades onde os serviços estão inseridos, promovendo diversas formas de aproximação interpessoal/ interprofissional de parcerias e de alcance interinstitucional.

Reato e Hirata (2019) apresentam o treinamento em medicina do adolescente, oferecido por meio da inserção da Disciplina de Hebiatria para o $5^{\circ}$. Ano da Graduação em Medicina e pelo Ciclo de Hebiatria para o $1^{\circ}$. Ano de Residência Médica em Pediatria, no Departamento de Medicina de Pediatria da Faculdade de Medicina do ABC, como forma de preparar os profissionais para atuação em equipe multiprofissional centrada no paciente adolescente. Para estes autores, dentro da estratégia de atendimento a essa população "reconhecida por sua vulnerabilidade", está a proposição da OMS para o ensino e capacitação de recursos humanos com a finalidade de melhor atendê-la.

No entanto, uma questão importante é o olhar da equipe para si mesma enquanto equipe, sem deixar de considerar que a competitividade entre os profissionais existe e pode ser positiva. E dar atenção às questões de poder, ética e habilidades de comunicação entre todos, com maturidade para lidar e solucionar os mais diferentes conflitos e situações:

É matar um leão por dia! O desafio do trabalho em equipe é que, quando a gente trabalha em equipe, que eu vejo querendo ou não, tem a briga dos egos profissionais, porque todo mundo quer mostrar sua competência na sua área. Também não vale a pena ficar numa equipe que não quer mostrar esse valor [...] conflitos têm, nós somos adultos, maduros. Mas tem que ter, e isso é sadio (Pedagoga2-Eq5).

Sim, é sadio e pode ser motivador dependendo da maturidade do grupo. $\mathrm{O}$ trabalho diferenciado, realizado em equipe e dedicado aos adolescentes não se pauta apenas em ações de curto prazo, de maneira que a constituição e o fortalecimento de uma equipe nem sempre acontece rapidamente. Os conflitos, presentes em todos os agrupamentos humanos, fazem parte do 
processo de atuação em conjunto e podem surgir de pontos de vista divergentes, falta de apoio técnico-operacional, dificuldades de adaptação individuais e coletivas, questões de poder, necessidade de reconhecimento, entre outros motivos (Schoen et al., 2019). Estas autoras afirmam que a confiança é um valor fundamental a ser cultivado entre os profissionais, uma vez que fortalece vínculos e reduz a vulnerabilidade diante de conflitos inerentes à atuação compartilhada.

Acho que tem dois principais. Primeiro a manutenção da equipe, a profissionalização da equipe, dentro da instituição. Outro grande desafio é dar sequência ao acompanhamento dessas crianças fora e depois da avaliação (Médico Pediatra-Eq5). - A gente nunca tem a equipe por completo. Então, eu acho que o mais difícil é isso, é você manter aquela equipe multidisciplinar (Neuropediatra-Especializado em Adolescentes-Eq2). - Eu acho que o nosso desafio é ter nesse momento de discutir nossos casos, por necessidade de agenda a gente perde um pouco desse momento da discussão, principalmente no ambulatório (Nutricionista-Eq4).

Em termos de constituição de equipe interdisciplinar de medicina e saúde do adolescente não há uma determinação que estabeleça como as equipes devem ser organizadas e quais as especialidades devem fazer parte da sua composição (Moraes \& Vitalle, 2021). Entende-se por equipe multiprofissional aquele grupo de profissionais das mais diversas formações que irão atuar em conjunto, estabelecendo um trabalho coletivo, de forma colaborativa, interdisciplinar e interprofissional. Desta forma, é importante a identificação da interdependência das diversas áreas de atuação e formação, individualmente, para estabelecer a junção e promover o crescimento no trabalho coletivo. Fundamental, portanto, o compartilhamento de conhecimentos, métodos e saberes individuais utilizados em prol dos adolescentes, famílias, professores e em todos os contextos em que estes sujeitos estejam inseridos (Silva e Schoen, 2019). Na contemporaneidade esta modalidade de trabalho exige a construção coletiva de uma relação recíproca, estabelecida entre todos os membros da equipe, onde a interação dos profissionais, sejam de que área for, se estabelece de modo contínuo, com base na afinidade e marcada por valores como cooperação, integração de saberes e práticas. Em síntese, as relações interpessoais são imprescindíveis para que a atuação das equipes opere de forma eficaz e efetiva. (Morais \& Vitalle, 2021). Neste sentido, observamos em nosso estudo que as equipes foram se constituindo com o propósito de efetivar uma atuação diferenciada, dirigida a um público-alvo que é o de adolescentes, cuja organização aconteceu conforme as necessidades de atuação foram surgindo.

Este estudo se pautou em um desenho qualitativo, cuja importância reside em apontar o significado de um determinado fenômeno, o que pode ser uma limitação no tocante a conhecer a magnitude do evento. Desta forma sugerimos a realização de novas pesquisas no tema proposto, com desenho de método misto, que para além de observar novos significados em novas categorias, apontem quantitativamente a amplitude, a extensão do evento. Deste modo, a amostragem determinada de forma probabilística, por meio de cálculo de tamanho de amostra, envolvendo equipes em todo o país, poderia fornecer indicadores relevantes do que acontece em todas as equipes que atuam com a saúde de adolescentes, tendo, portanto, validade interna e externa e possibilitaria assim, direcionar ações e estratégias apropriadas para corrigir itinerários e além de reforçar e disseminar os modelos mais adequados já existentes e propor novos modelos de atuação. A combinação de entrevistas abertas e fechadas (escalas, cheklist, survey, por ex.) com grupos focais, poderá ser auxiliar valioso na obtenção de informações mais precisas, mais objetivas que poderão complementar e mostrar outros ângulos de visão da atuação em equipe multiprofissional de medicina e saúde de adolescentes. Outro ponto a ser levado em conta, que pode trazer novas contribuições para o entendimento da formação das equipes e quais mudanças serão necessárias propor é considerar, além da percepção dos profissionais como membros de equipes, o olhar dos adolescentes sobre as dificuldades e os desafios encontrados no dia a dia nos serviços que frequentam. 


\section{Considerações Finais}

Em relação ao acesso aos serviços de medicina e saúde do adolescente, encontramos alguns obstáculos iniciais quando, pela busca via internet, nos deparamos com informações de contato na maioria dos sites dos HU e Universidades $(e-$ mails e telefones) não estavam atualizadas e objetivas; e quando as ligações eram atendidas, iam sendo transferidas e raramente se completavam.

Os obstáculos foram superados e o nosso deslocamento, para o encontro com os profissionais que aceitaram ser entrevistados, mostrou-se favorável para conhecermos de perto a realidade desses serviços oferecidos em cinco diferentes localizações no país, o que trouxe maior enriquecimento ao estudo e à nossa própria formação. As falas são representadas no território nacional, entra no cenário da pesquisa com a descrição dos cinco serviços que não tem diferença da fala do indivíduo que está em Brasília e do que está em Florianópolis, por exemplo.

Dentre os principais motivos de consulta encontramos as queixas clínicas de saúde geral, bem como de ordem nutricional, postural, saúde mental, questões de gênero, questões escolares, violências de diferentes formas, incluindo casos de bullying, doenças crônicas entre outros. A questão escolar mostrou-se como uma das grandes preocupações dos profissionais.

A sobrecarga de trabalho e a dificuldade para manterem as equipes estão entre os desafios encontrados.

Encontramos profissionais dedicados ao atendimento dos adolescentes, com acolhimento, cuidado e alto nível de conhecimento e experiência em suas áreas de atuação.

De acordo com os entrevistados o trabalho diferenciado, realizado em equipe, dedicado aos adolescentes não se pauta apenas em ações de curto prazo, de maneira que a constituição e o fortalecimento de uma equipe nem sempre acontece rapidamente. O esforço é permanente, mas, como foi muito bem colocado nos relatos, sem políticas públicas não há garantias de direitos. Elas são fundamentais para que o atendimento integral à saúde dos adolescentes se efetive de forma ampla em toda a rede pública de atendimento em saúde. Mesmo não tendo a intencionalidade de buscar modelos ou propor modelos encontramos propostas sugeridas pelas próprias equipes. No tocante à formação dos profissionais emergem questões de comunicação, teóricas e práticas, histórico-culturais. Estas questões podem ser auxiliares valiosos na promoção da mudança por meio de ações educativas e de ensino que a subsidiem.

\section{Referências}

Azevedo, A. E. B. I. (2019). Os Desafios do Atendimento Ambulatorial: das questões locais às políticas Públicas de Saúde. In: Vitalle, M.S.S., Silva, F.C., Pereira, A. M. L., Weiler, R. M. E., Niskier, S. R., Schoen, T. H. (Org.). Medicina do Adolescente: fundamentos e prática. Atheneu, p. 19-25.

Bardin, L. (2016). Análise de Conteúdo. Edições 70.

Brasil. Estatuto da Criança e do Adolescente (ECA). (1990). estatuto-da-crianca-e-do-adolescente-versao-2019.pdf. Lei 8069, de 13 de julho de 1990. https://www.gov.br/mdh/pt-br/centrais-de-conteudo/crianca-e-adolescente/estatuto-da-crianca-e-do-adolescente-versao-2019.pdf

Brasil. Estatuto da Juventude (2013). https://www2.senado.leg.br/bdsf/bitstream/handle/id/509232/001032616.pdf

Brasil. Ministério da Saúde. (2018). Secretaria de Atenção à Saúde. Departamento de Ações Programáticas e Estratégica. Proteger e cuidar da saúde do adolescente na atenção básica. $2^{\mathrm{a}}$ ed, Brasília, D.F., 235p.

Brasil. Ministério da Saúde. Secretaria de Atenção em Saúde. Departamento de Ações Programáticas Estratégicas. (2010). Diretrizes nacionais para a atenção integral à saúde de adolescentes e jovens na promoção, proteção e recuperação da saúde. / Brasília: Ministério da Saúde, 2010. 132 p.: il. - (Série A. Normas e Manuais Técnicos) - ESF

Brasil. Ministério da Saúde. Sistema Único de Saúde (SUS). (http://bvsms.saude.gov.br/ultimas-noticias/3295-lei-n-8080-30-anos-de-criacao-do-sistemaunico-de-saude-

sus\#: :text=Alta $\% 20$ Complexidade,Lei $\% 20$ n\%C2\%BA\%208080\%3A\%2030\%20anos\%20de $\% 20$ cria\%C3\%A7\%C3\%A3o,Sistema\%20\%C3\%9Anico\%20de \%20Sa\%C3\%BAde\%20(SUS)\&text=Em\%2019\%2F9\%2F1990\%20foi,\%C3\%9Anico\%20de\%20Sa\%C3\%BAde\%20(SUS)).

Brasil. OMS. Diretrizes Nacionais de Atenção à Saúde de Adolescentes e Jovens. (2010). https://bvsms.saude.gov.br/bvs/publicacoes/diretrizes_nacionais_atencao_saude_adolescentes_jovens_promocao_saude.pdf 
Costa J. R. \& Couto, M.T. (2015). Geração e categorias geracionais nas pesquisas sobre saúde e gênero no Brasil. Saúde \& Sociedade. 24(4), $1299-1315$.

Diário Oficial da União. Conselho Federal de Medicina (CFM). https://www.semesp.org.br/wp-content/uploads/2021/09/resolucoes-cnrm-n-33-a-n-59-de-2de-setembro-de-2021.pdf

Diretrizes Curriculares Nacionais do Curso de Graduação em Medicina (2014). http://portal.mec.gov.br/index.php? option=com_docman\&view=download\&alias=15874-rces003-14\&category_slug=junho-2014-pdf\&Itemid=30192

Eisenstein, E. (2018). Medicina \& Saúde de Adolescentes: o octaedro de ações. Adolesc. Saúde, 15, supl. 1, 21-33.

Fernandes, E. S. F. \& Santos, A.M. (2020). Desencontros entre formação profissional e necessidades de cuidado aos adolescentes na Atenção Básica à Saúde. Rev Interface, 24, e190049.

Gil, A. C. (2017). Como elaborar projetos de pesquisa. Atlas, 6. Ed., 192p.

Jager, M. E., Batista, F. A., Cláudia, M. P., Samara, S.S. \& Dias, A.C.G. (2014). O Adolescente no Contexto da Saúde Pública Brasileira: Reflexões sobre o PROSAD. Psicologia em Estudo, 19 (2), 211-221.

Juckowsky Filho, D. (1991). A Abordagem Multidisciplinar do Adolescente. In: Markaroun, M.F., Souza, R.P., Cruz, A.R. (Org.) Tratado de Adolescência Um Estudo Multidisciplinar. Cultura Médica, p. 233-238.

Moraes, S. P. \& Vitalle M.S.S. (2021). Educação em Saúde e direitos sexuais e reprodutivos na adolescência. e-Book. Uniedusul. ISBN: 978-65-86010-89-3. Doi. $10.51324 / 86010893$.

Moreira, D. A. (2002). O Método Fenomenológico na Pesquisa. Pioneira/Thomson, 152p.

Organização Panamericada da Saúde. OPAS. (2019). 57o CONSELHO DIRETOR 71a SESSÃO DO COMITÊ REGIONAL DA OMS PARA AS AMÉRICAS Washington, D. C., EUA, de 30 de setembro a 4 de outubro de 2019. Estratégia e plano de ação para a saúde do adolescente e do jovem: relatório final. https://iris.paho.org/bitstream/handle/10665.2/51633/CD57-INF-8-p.pdf?sequence=3\&isAllowed=y

Organização Mundial da Saúde. OMS. (1977). Necessidades de la salud de los adolescentes. Informe de um comité de expertos de la OMS. Informe técnico ${ }^{\circ}$ 609. OMS. Genebra: Organização Mundial da Saúde (OMS).

Paul, P. (2013). Saúde e Transdisciplinaridade: a importância da subjetividade nos cuidados médicos. Edusp, 288p.

Peres F. \& Rosenburg, C. (1998). Desvelando a concepção de adolescência / adolescente presente no discurso da saúde pública. Rev Saúde e Sociedade, 7 (1), 5386.

Queiroz, L. B., Ayres, J. R. C. M., Saito, M. I. \& Mota, A. (2013). Aspectos históricos da institucionalização da atenção à saúde do adolescente no estado de São Paulo, 1970-1990. Revista: História, Ciência e Saúde, 20 (1), 49-66.

Reato, L. F. N. \& Hirata, A. M. (2019). Fundamentos do Processo Ensino-Aprendizagem da Medicina de Adolescente. In: Vitalle, M.S.S., Silva, F.C., Pereira, A. M. L., Weiler, R. M. E., Niskier, S. R., Schoen, T.H. (Org.). Medicina do Adolescente: fundamentos e prática. Atheneu, p.3-7.

Schoen, T. H. \& Marteleto, M. R. F. (2019). Aspectos Pedagógicos. In: Vitalle, M.S.S., Silva, F.C., Pereira, A.M.L., Weiler, R. M. E., Niskier, S. R., Schoen, T.H. (Org.). Medicina do Adolescente: fundamentos e prática/editores. Atheneu, p. 305-310.

Schoen, T.H., Silva, D. A., Silva, F. C. \& Vitalle, M. S. S. (2019). Abordagem Transdisciplinar no atendimento ao adolescente frente às suas transformações. In: Takaoka, L.A.M.V., Coutinho, L., Weiler, R. M. E. (Org.). Odontopediatria: transdisciplinaridade na saúde e na educação da criança e do adolescente. BOK2., p. 101-106

Silva, D. A. (2017). Equipes de Medicina do Adolescente: constituição, funcionamento, valores e prática inter e transdisciplinar. (Tese de Doutorado). Universidade Federal de São Paulo. São Paulo, SP, Brasil. https://repositorio.unifesp.br/handle/11600/50850

Silva, D. A. \& Schoen, T. H. (2019). O Trabalho em Equipe Multiprofissional. In: Vitalle, M. S. S., Silva, F. C., Pereira, A. M. L., Weiler, R. M. E., Niskier, S. R., Schoen, T.H. (Org.). Medicina do Adolescente: fundamentos e prática/editores. Atheneu, p. 601-607. 